\title{
BIFURCATION FOR QUASILINEAR ELLIPTIC SINGULAR BVP
}

\author{
DAVID ARCOYA, JOSÉ CARMONA, AND PEDRO J. MARTÍNEZ-APARICIO
}

Dedicado a la memoria de nuestra amiga Fuensanta Andreu

\begin{abstract}
For a continuous function $g \geq 0$ on $(0,+\infty)$ (which may be singular at zero), we confront a quasilinear elliptic differential operator with natural growth in $\nabla u,-\Delta u+g(u)|\nabla u|^{2}$, with a power type nonlinearity, $\lambda u^{p}+f_{0}(x)$. The range of values of the parameter $\lambda$ for which the associated homogeneous Dirichlet boundary value problem admits positive solutions depends on the behavior of $g$ and on the exponent $p$. Using bifurcations techniques we deduce sufficient conditions for the boundedness or unboundedness of the cited range.
\end{abstract}

\section{INTRODUCTION}

Let $\Omega$ be an open and bounded subset of $\mathbb{R}^{N}(N \geq 3), \lambda \geq 0,0 \leq p<\frac{N+2}{N-2}$ and $0 \leq f_{0} \in L^{\frac{2 N}{N+2}}(\Omega)$. Consider the boundary value problem

$$
\begin{cases}-\Delta u+g(u)|\nabla u|^{2}=\lambda u^{p}+f_{0}(x) & \text { in } \Omega, \\ u=0 & \text { on } \partial \Omega,\end{cases}
$$

for a suitable non negative continuous function $g:(0,+\infty) \rightarrow[0,+\infty)$. We say that $u \in H_{0}^{1}(\Omega)$ is a positive solution for (1.1) if $u>0$ a.e. $x \in \Omega, g(u)|\nabla u|^{2} \in L^{1}(\Omega)$ and

$$
\int_{\Omega} \nabla u \nabla \varphi+\int_{\Omega} g(u)|\nabla u|^{2} \varphi=\lambda \int_{\Omega} u^{p} \varphi+\int_{\Omega} f_{0} \varphi,
$$

for every $\varphi \in H_{0}^{1}(\Omega) \cap L^{\infty}(\Omega)$. Notice that the differential operator appearing in (1.1) contains a lower order term with quadratic growth in the gradient. From the pioneering works by Boccardo, Murat and Puel $[12,13]$ this kind of quasilinear operators with $g$ a continuous function in $[0,+\infty)$ has been extensively studied, especially if $\lambda=0$ in the right hand side. More recently and also for $\lambda=0$, the case of a function $g$ with a singularity at zero has been studied in $[5,6,8]$.

Observe that, in our case, the right hand side of the equation in (1.1) is linear iff $p=1$. This case has also been recently studied by Abdellaoui, Peral and Primo [1] for $g \equiv 1$. They prove existence of a positive solution for every $\lambda \in[0,+\infty)$ provided that $f_{0} \gtreqless 0$. Compare this result with the semilinear case $(g \equiv 0)$ where existence of positive solution requires $\lambda$ being smaller than the first eigenvalue $\mu_{1}$ of the Laplacian operator with zero Dirichlet boundary conditions. In this way, the authors stressed that the quadratic term in the gradient produces a strong regularizing effect and break down any resonance effect of the linear zero-order term.

Research supported by MICINN Ministerio de Ciencia e Innovación (Spain) MTM2009-10878 and Junta de Andalucia FQM-116 and FQM-194. 
To our knowledge, the case in which the (non-variational) differential operator (with $g$ a continuous function in $[0,+\infty)$ ) is faced up to a true nonlinear right hand side has been less studied [17, 19]. See the works by Arcoya and Boccardo [4], Canino [15] and the references therein for the case of variational differential operators. See also the works by Andreu, Boccardo, Orsina and Segura de León [2], Andreu, Segura de León and Toledo [3] and Boccardo, Orsina and Porzio [14] for related parabolic problems. If $f_{0} \equiv 0$ and $p>1$, it is shown by Orsina and Puel [17] that the behavior of $g$ at infinity has also an effect on the positive solution set. Specifically, the authors use a suitable change of variables which reduces the quasilinear equation to a semilinear one and thus they prove that if $g \in L^{1}(0,+\infty)$ then there exists positive solution for every $\lambda>0$, while if $g(t) t \geq q>p$ for $t>>1$, then there exists a positive solution for $\lambda>0$ large enough and no positive solution if $\lambda>0$ is sufficiently small.

One of the main purposes of this paper is to provide a common framework (based on topological methods) which handles the previous results and helps us to understand the true role of the different hypotheses imposed on the behavior of the non linearity $g$, revealing the different effects that take place in the solution set according as $p=1$ or $p \neq 1, f_{0} \equiv 0$ or $f_{0} \gtreqless 0$. As a particular case of our results we will show that even in the case $p=1$, the behavior of $g$ at infinity has a role in the solution set, for example, no "strong regularizing effect" is obtained for functions $g$ which are sufficiently small at infinity. This is not the case if $p<1$ where the positive solution set for $g \not \equiv 0$ behaves like in the semilinear case $g \equiv 0$. Finally, if $p>1$ and $f_{0} \not \equiv 0$, the behavior of $g$ at infinity is again determinant to have some kind of "regularizing effect".

Furthermore, another of the our goals is to extend the above results to handle, in addition to the case of continuous functions $g$ on $[0,+\infty)$, the case of functions $g:(0,+\infty) \rightarrow[0,+\infty)$ which may be singular at zero. Specifically, to unify both cases: the continuous and the singular ones, we assume in the whole paper the following hypothesis:

(G) Either the function $g \geq 0$ is continuous in $[0,+\infty)$ or $g \geq 0$ is continuous in $(0,+\infty)$, decreasing and integrable in a neighborhood of zero with $\lim _{s \rightarrow 0} g(s)=+\infty$.

In this way, we can consider the operator $K: \mathbb{R} \times H_{0}^{1}(\Omega) \rightarrow H_{0}^{1}(\Omega)$ by defining, for every $\lambda \in \mathbb{R}$ and for every $w \in H_{0}^{1}(\Omega), K(\lambda, w)$ as the unique solution $u$ in $H_{0}^{1}(\Omega)$ of the problem

$$
\begin{cases}-\Delta u+g(u)|\nabla u|^{2}=\lambda^{+} w^{+}(x)^{p}+f_{0}(x) & \text { in } \Omega, \\ u=0 & \text { on } \partial \Omega .\end{cases}
$$

Indeed, in the case that $g$ is singular at zero with $0 \lesseqgtr f_{0} \in L^{2 N /(N+2)}(\Omega)$ the existence is due to [8] and the uniqueness to [6]. Moreover, if $g$ is continuous at zero, the existence $([12])$ and uniqueness $([6])$ results remain also true in the case $f_{0} \equiv 0$. With this notation, (1.1) can be rewritten as a fixed point problem, namely,

$$
u=K_{\lambda}(u),
$$

with $K_{\lambda}(u)=K(\lambda, u)$.

Compare this approach with this one in the work by Ruiz and Suárez [19], for $g \equiv 1$ and a logistic nonlinearity, where the authors cleverly combine regularity in $C^{1}(\Omega)$ with the properties of the inverse $(-\Delta)^{-1}$ of the Laplacian operator in $C(\Omega)$ 
in order to use bifurcation techniques. Unfortunately, this idea does not work in the case $g$ singular at zero by the lack of regularity.

We prove the compactness of $K$ in Section 2 which allows us to apply the LeraySchauder degree techniques to study the existence of "continua of solutions" of (1.1), i.e. connected and closed subsets in the solution set

$$
\left\{(\lambda, u) \in \mathbb{R} \times H_{0}^{1}(\Omega): u=K_{\lambda}(u)\right\} .
$$

It has been remarked that the computation of the degree of the operators $K_{\lambda}$ will be carried out by constructing suitable homotopies with a linear operator (the Laplacian one), reducing in this way the study of the quasilinear problem (1.1) to a linear one.

It is in this unified functional framework that we work and we obtain our results that we present here for a simple nonlinearity $f(\lambda, x, s)=\lambda s^{p}$ and $g(x, s)$ independent on $x \in \Omega$, although most of them hold true for a suitable perturbation of them. To be more specific, in the case $p=1$ we give sufficient conditions on $g$ to have, as in the semilinear case, existence of solution for a bounded interval of values of the parameter $\lambda$. Roughly speaking, those conditions are related to how far from zero we can take $g$ in order to remain true the "semilinear type" result. On the other hand, we also prove a regularizing effect, that is, existence of solution in an unbounded interval of values for the parameter $\lambda$ provided that $g$ is sufficiently far from zero.

Theorem 1.1. Assume $p=1,0 \lessgtr f_{0} \in L^{\frac{2 N}{N+2}}(\Omega)$ and suppose that condition $(G)$ holds.

(1) (No regularizing effect) If there are $s_{0}, \delta_{0}>0$ such that

$$
\frac{s}{\int_{0}^{s} e^{\int_{r}^{s} g(t) d t} d r} \geq \delta_{0}, \quad \forall s>s_{0},
$$

then there exist $\lambda^{*}, \lambda_{*}>0$ such that (1.1) has no positive solution for $\lambda>\lambda^{*}$ and admits a positive solution for every $\lambda \in\left[0, \lambda_{*}\right)$.

(2) (Regularizing effect) If there exist $s_{1}, c>0$, and $\gamma<1$ such that

$$
g(s) \geq \frac{c}{s^{\gamma}}, \quad \forall s \geq s_{1},
$$

then (1.1) admits a positive solution for every $\lambda \in[0,+\infty)$.

Observe that there is a gap between both conditions (1.3) and (1.4) since, for instance, the function $g(s)=\frac{c}{s+1}$ with $c \geq 1$ satisfies neither (1.3) nor (1.4).

In the case $0 \leq p<1$ we show that the behavior of $g$ at infinity has no influence in the solution set since there exists a positive solution for every $\lambda \in(0,+\infty)$.

Theorem 1.2. If $0 \leq p<1,0 \leq f_{0} \in L^{\frac{2 N}{N+2}}(\Omega)$ and hypothesis $(G)$ is satisfied, then (1.1) admits a positive solution for every $\lambda \in(0,+\infty)$.

With respect to the case $p>1$, we have the following result.

Theorem 1.3. Consider $p>1,0 \lesseqgtr f_{0} \in L^{\frac{2 N}{N+2}}(\Omega)$ and assume that hypothesis $(G)$ is satisfied.

i) If condition (1.4) holds with $0 \leq \gamma<2-p$, then problem (1.1) admits a positive solution for every $\lambda \in[0,+\infty)$. 
ii) If there are $s_{0}, \delta_{0}>0$ such that

$$
\frac{s^{p}}{\int_{0}^{s} e^{\int_{r}^{s} g(t) d t} d r} \geq \delta_{0}, \quad \forall s>s_{0},
$$

then there exist $\lambda^{*}, \lambda_{*}>0$ such that (1.1) admits a positive solution for every $\lambda \in\left[0, \lambda_{*}\right)$ and admits no positive solution for $\lambda>\lambda^{*}$.

If $g$ is continuous in zero, we can handle the case $f_{0} \equiv 0$ and to obtain the following result.

Theorem 1.4. Assume $f_{0} \equiv 0$ and suppose that $g \geq 0$ is continuous in the interval $[0,+\infty)$.

(1) (No regularizing effect) If $p=1$ and (1.3) holds, then there exist $\lambda^{*}, \lambda_{*}>\mu_{1}$ such that (1.1) has no positive solution for $\lambda>\lambda^{*}$ and admits a positive solution for every $\lambda \in\left(\mu_{1}, \lambda_{*}\right)$

(2) (Regularizing effect) If $p=1$ and (1.4) holds, then (1.1) admits a positive solution if and only if $\lambda>\mu_{1}$.

(3) If $0 \leq p<1$, then (1.1) admits a positive solution for every $\lambda \in(0,+\infty)$.

(4) If $p>1$ and there is a continuous non positive function $h \in L^{1}(0,+\infty)$ such that

$$
g(s) \geq h(s)+\frac{p}{s}, \quad \forall s \geq 1,
$$

then there exists $\lambda^{*}>0$ such that (1.1) has no solution for $\lambda<\lambda^{*}$.

We remark that cases (1)-(3) of the above result are new, while (4) is a slight improvement of [17] where it is required $g(s)>q / s$ for large $s$, with $q>p$.

\section{Preliminaries}

For every $s \in \mathbb{R}$ we consider the positive and negative parts given by $s^{+}=$ $\max \{s, 0\}$ and $s^{-}=\min \{s, 0\}$. We denote by $T_{k}$ and $G_{k}$ the usual truncature functions given by $T_{k}(s)=\min \left\{k, s^{+}\right\}+\max \left\{-k, s^{-}\right\}$and $G_{k}(s)=s-T_{k}(s)$, for every $s \in \mathbb{R}$. For $1 \leq p \leq+\infty,\|u\|_{p}$ is the usual norm of a function $u \in L^{p}(\Omega)$. We equipped the standard Sobolev space $H_{0}^{1}(\Omega)$ with the norm $\|u\|=\left(\int_{\Omega}|\nabla u|^{2}\right)^{1 / 2}$. We also denote by $\mathcal{S}=\sup \left\{\|u\|_{2^{*}}:\|u\|=1\right\}$ the Sobolev embedding constant $\left(2^{*}=2 N /(N-2)\right)$.

Observe that our definition of solution of (1.1) includes the integrability of $g(u)|\nabla u|^{2}$. We will see in the following result that a consequence is the integrability of $g(u)|\nabla u|^{2} \varphi$ for every $\varphi \in H_{0}^{1}(\Omega)$.

Lemma 2.1. If $0<u \in H_{0}^{1}(\Omega)$ is a solution for (1.1), then $g(u)|\nabla u|^{2} \varphi$ is integrable in $\Omega$ for every $\varphi \in H_{0}^{1}(\Omega)$ and, in particular, it holds

$$
\int_{\Omega} \nabla u \nabla \varphi+\int_{\Omega} g(u)|\nabla u|^{2} \varphi=\lambda \int_{\Omega} u^{p} \varphi+\int_{\Omega} f_{0} \varphi, \quad \forall \varphi \in H_{0}^{1}(\Omega) .
$$

Proof. Since $p \leq 2^{*}-1$ observe that $u^{p}, u^{p} \varphi \in L^{1}(\Omega)$ for every $\varphi \in H_{0}^{1}(\Omega)$. Even more, taking $T_{k}\left(\varphi^{+}\right)$as test function in (1.2), and using the Hölder inequality we 
have

$$
\begin{aligned}
\int_{\Omega} g(u)|\nabla u|^{2} T_{k}\left(\varphi^{+}\right) & =-\int_{\Omega} \nabla u \nabla T_{k}\left(\varphi^{+}\right)+\int_{\Omega}\left(\lambda u^{p}+f_{0}\right) T_{k}\left(\varphi^{+}\right) \\
& \leq\|u\|\left\|\varphi^{+}\right\|+\int_{\Omega}\left(\lambda u^{p}+f_{0}\right) \varphi^{+} .
\end{aligned}
$$

Taking limit as $k \rightarrow \infty$ and using Fatou Lemma, we deduce that $g(u)|\nabla u|^{2} \varphi^{+} \in$ $L^{1}(\Omega)$ with

$$
\int_{\Omega} g(u)|\nabla u|^{2} \varphi^{+} \leq\|u\|\left\|\varphi^{+}\right\|+\int_{\Omega}\left(\lambda u^{p}+f_{0}\right) \varphi^{+} .
$$

Similarly, taking $T_{k}\left(-\varphi^{-}\right)$as test function in (1.2) we obtain that $g(u)|\nabla u|^{2} \varphi^{-} \in$ $L^{1}(\Omega)$ and

$$
-\int_{\Omega} g(u)|\nabla u|^{2} \varphi^{-} \leq\|u\|\left\|\varphi^{-}\right\|-\int_{\Omega}\left(\lambda u^{p}+f_{0}\right) \varphi^{-} .
$$

In particular, $g(u)|\nabla u|^{2} \varphi \in L^{1}(\Omega)$ and

$$
\int_{\Omega} g(u)|\nabla u|^{2}|\varphi| \leq\|u\|\|\varphi\|+\int_{\Omega}\left(\lambda u^{p}+f_{0}\right)|\varphi| .
$$

Finally, a density argument concludes (2.1).

Remark 2.2. The above lemma can be improved by showing that if $0<u \in H_{0}^{1}(\Omega)$ is a solution of (1.1), then $g(u)|\nabla u|^{2} \varphi \in L^{1}(\Omega)$ for every $\varphi \in H^{1}(\Omega)$. Indeed, arguing as in the proof of the lemma, this is deduced by taking now $\frac{T_{\varepsilon}(u)}{\varepsilon} T_{1 / \varepsilon}\left(\varphi^{ \pm}\right)$ as test function in (1.1).

The following lemma concerning with the regularity of the solutions will be useful in the sequel. It can be easily deduced by using the Stampacchia technique [20].

Lemma 2.3. Assume that $f_{0} \in L^{q}(\Omega)$ with $q>\frac{N}{2}$ and that condition $(G)$ is satisfied. If $u \in H_{0}^{1}(\Omega)$ is a solution for (1.1), then $u$ belongs to $L^{\infty}(\Omega)$. Moreover, in the case $f_{0} \equiv 0$ and $p \leq 1$, there exists a positive constant $\alpha$ such that

$$
\|u\|_{\infty} \leq \alpha\|u\|^{t},
$$

where $t=\frac{2^{*} p}{r-p r+p 2^{*}}$ with $r$ any number greater than $\max \left\{N p / 2,2^{*}\right\}$.

Now we give sufficient conditions to assure that problem (1.1) satisfies the uniform strong maximum principle (USMP), that is, for every $\omega \subset \subset \Omega$ there exists a positive constant (independent from $\lambda$ ) which is a lower bound in $\omega$ of any solution of (1.1).

Proposition 2.4. Suppose that $0 \lesseqgtr f_{0}$ and that hypothesis $(G)$ holds. Then for every $\omega \subset \subset \Omega$ there exists $L_{\omega}>0$ such that,

$$
u(x) \geq L_{\omega}, \quad \text { a.e. } \quad x \in \omega,
$$

for every supersolution $u \in H_{0}^{1}(\Omega)$ of (1.1) (with $\lambda$ any positive constant).

Proof. Taking into account that $\lambda s^{p}+f_{0}(x) \geq T_{1}\left(f_{0}(x)\right)$, for all $s \geq 0$, every solution $u \in H_{0}^{1}(\Omega)$ is a supersolution for the problem

$$
\begin{cases}-\Delta v+g(v)|\nabla v|^{2}=T_{1}\left(f_{0}(x)\right) & \text { in } \Omega, \\ v=0 & \text { on } \partial \Omega .\end{cases}
$$


Since $g$ is integrable in a neighborhood of zero, this problem has a unique continuous solution $v \in H_{0}^{1}(\Omega) \cap C(\Omega)$ (see [8] for existence and [6] for uniqueness). Using that $v \in C(\Omega)$ and $v>0$ in $\Omega$, if $\omega \subset \subset \Omega$ we infer the existence of $L_{\omega}>0$ such that $v(x) \geq \min _{\omega} v=L_{\omega}$. By the comparison principle in [6] we deduce that $u \geq v \geq L_{\omega}$ and the proof is concluded.

The following result will be concerned with the compactness for the operator $K(\lambda, w)$.

Proposition 2.5. Assume that hypothesis $(G)$ holds. If the sequences $t_{n} \in[0,1]$ and $\lambda_{n}>0$ are convergent, respectively, to $t^{*}$ and $\lambda$, and $w_{n}$ is $H_{0}^{1}(\Omega)$-weakly convergent to $w$, then the sequence of the unique solution $u_{n} \in H_{0}^{1}(\Omega)$ of

$$
\begin{cases}-\Delta u_{n}+t_{n} g\left(u_{n}\right)\left|\nabla u_{n}\right|^{2}=\lambda_{n} w_{n}^{+}(x)^{p}+f_{0}(x) & \text { in } \Omega, \\ u_{n}=0 & \text { on } \partial \Omega,\end{cases}
$$

is strongly convergent in $H_{0}^{1}(\Omega)$ to the solution $u$ of

$$
\begin{cases}-\Delta u+t^{*} g(u)|\nabla u|^{2}=\lambda w^{+}(x)^{p}+f_{0}(x) & \text { in } \Omega, \\ u=0 & \text { on } \partial \Omega .\end{cases}
$$

Remark 2.6. We point out that in order to apply [6] for assuring the uniqueness of solution for problems (2.3) and (2.4), we have to impose that $t_{n} g(s)$ and $t^{*} g(s)$ are integrable in a neighborhood of zero.

Proof. If $g \geq 0$ is continuous in $[0,+\infty)$ and the datum $0 \leq f_{0} \in L^{\frac{2 N}{N+2}}(\Omega)$, then the proof follows by [7]. Here, we give a proof valid also in the case of a singular $g$, that is, $g \geq 0$ is continuous in $(0,+\infty)$, decreasing and integrable in a neighborhood of zero with $\lim _{s \rightarrow 0} g(s)=+\infty$ and the datum $0 \leqq f_{0} \in L^{\frac{2 N}{N+2}}(\Omega)$. It suffices to prove that every subsequence of $\left\{u_{n}\right\}$ possesses a subsequence converging to the unique solution $u \in H_{0}^{1}(\Omega)$ of (2.4). First we prove that $u_{n}$ is bounded in $H_{0}^{1}(\Omega)$. Indeed, choosing $u_{n}$ as test function in (2.3), using that $g$ is nonnegative and Hölder and Sobolev inequalities and taking into account that $p \frac{2 N}{N+2} \leq\left(2^{*}-1\right) \frac{2 N}{N+2}=2^{*}$ we have

$$
\left\|u_{n}\right\| \leq \mathcal{S}\left(\lambda_{n} \mathcal{S}^{p}\left\|w_{n}\right\|^{p}|\Omega|^{1-\frac{p}{2^{*}}}+\left\|f_{0}\right\|_{2 N /(N+2)}\right) .
$$

Thus the boundedness of $\left\|w_{n}\right\|$ and $\lambda_{n}$ implies that $u_{n}$ is bounded in $H_{0}^{1}(\Omega)$. Therefore, there exists $\bar{u} \in H_{0}^{1}(\Omega)$ such that, up to a subsequence, $u_{n}$ converges to $\bar{u}$ weakly in $H_{0}^{1}(\Omega)$, strongly in $L^{2}(\Omega)$ and almost everywhere in $\Omega$.

Moreover, choosing $\frac{1}{\varepsilon} T_{\varepsilon}\left(u_{n}\right)$ as test function in (2.3) and dropping some positive terms we deduce that

$$
\int_{\Omega} \frac{T_{\varepsilon}\left(u_{n}\right)}{\varepsilon} t_{n} g\left(u_{n}\right)\left|\nabla u_{n}\right|^{2} \leq \lambda_{n} \int_{\Omega}\left(w_{n}^{+}\right)^{p}+\int_{\Omega} f_{0} .
$$

If we take the limit as $\varepsilon$ tends to zero, we get from Fatou lemma

$$
\int_{\Omega} t_{n} g\left(u_{n}\right)\left|\nabla u_{n}\right|^{2} \leq \lambda_{n} \int_{\Omega}\left(w_{n}^{+}\right)^{p}+\int_{\Omega} f_{0} .
$$

Therefore, using again $\left\|w_{n}\right\|$ and $\lambda_{n}$ are bounded sequences we have that the sequence $t_{n} g\left(u_{n}\right)\left|\nabla u_{n}\right|^{2}$ is bounded in $L^{1}(\Omega)$.

Since $\lambda_{n}\left(w_{n}(x)^{+}\right)^{p}+f_{0}(x)-t_{n} g\left(u_{n}\right)\left|\nabla u_{n}\right|^{2}$ is bounded in $L^{1}(\Omega)$, we can apply Lemma 1 of [9] (see also [11]) to deduce that, up to (not relabeled) subsequences, 
$\nabla u_{n} \rightarrow \nabla \bar{u}$ a.e. in $\Omega$. Even more, using Proposition 2.4 we can argue as in [5] to deduce that $u_{n} \rightarrow \bar{u}$ in $H_{\mathrm{loc}}^{1}(\Omega)$ and $\bar{u}$ is a solution for (2.4) and thus, by the uniqueness [6], $\bar{u}=u$.

If we fix now $k \in \mathbb{R}$, we observe that $u_{n}=G_{k}\left(u_{n}\right)+T_{k}\left(u_{n}\right)$ and thus the strong convergence of $u_{n}$ in $H_{0}^{1}(\Omega)$ is stated provided that we show the strong convergence of $G_{k}\left(u_{n}\right)$ and $T_{k}\left(u_{n}\right)$. This is done in two steps:

Step 1. $G_{k}\left(u_{n}\right)$ is strongly convergent to $G_{k}(u)$ in $H_{0}^{1}(\Omega)$. Indeed, taking $G_{k_{0}+k}\left(u_{n}\right)$ as test function in $(2.3)$ we have

$$
\int_{\Omega}\left|\nabla G_{k_{0}+k}\left(u_{n}\right)\right|^{2} \leq \frac{\mathcal{S}^{2}}{\alpha^{2}}\left(\int_{\left\{u_{n} \geq k_{0}\right\}}\left(\lambda_{n}\left(w_{n}^{+}\right)^{p}+f_{0}\right)^{\frac{2 N}{N+2}}\right)^{1+2 / N} .
$$

Since $w_{n}$ is strongly convergent in $L^{\frac{2 N p}{N+2}}(\Omega), \lambda_{n}$ is bounded and $f_{0} \in L^{\frac{2 N}{N+2}}(\Omega)$, the right-hand side of the last inequality tends uniformly in $n$ to zero as $k_{0}$ diverges. Therefore, we deduce that for every $\varepsilon>0$, it is possible to choose $k_{0}>0$ (depending on $\varepsilon$ ) such that

$$
\left\|G_{k_{0}}\left(G_{k}\left(u_{n}\right)\right)\right\|+\left\|G_{k_{0}}\left(G_{k}(u)\right)\right\| \leq \varepsilon, \quad \forall n \in \mathbb{N} .
$$

Observe that

$$
\begin{aligned}
\left\|G_{k}\left(u_{n}\right)-G_{k}(u)\right\| \leq & \left\|T_{k_{0}}\left(G_{k}\left(u_{n}\right)\right)-T_{k_{0}}\left(G_{k}(u)\right)\right\| \\
& +\left\|G_{k_{0}}\left(G_{k}\left(u_{n}\right)\right)\right\|+\left\|G_{k_{0}}\left(G_{k}(u)\right)\right\| \\
\leq & \left\|T_{k_{0}}\left(G_{k}\left(u_{n}\right)\right)-T_{k_{0}}\left(G_{k}(u)\right)\right\|+\varepsilon .
\end{aligned}
$$

Therefore, to show the strong convergence of $G_{k}\left(u_{n}\right)$ to $G_{k}(u)$ in $H_{0}^{1}(\Omega)$ (as $n$ goes to infinity), it only remains to prove that $T_{k_{0}}\left(G_{k}\left(u_{n}\right)\right)$ is strongly convergent to $T_{k_{0}}\left(G_{k}(u)\right)$ in $H_{0}^{1}(\Omega)$. In order to make that, we argue as in [10]. We denote the sequence $T_{k_{0}}\left(G_{k}\left(u_{n}\right)\right)-T_{k_{0}}\left(G_{k}(u)\right)$ by $\varpi_{n}$ and set $\varphi_{\gamma}(s)=s e^{\gamma s^{2}}$ with $\gamma>c^{2}$, where $c=\max \left\{g(s): k \leq s \leq k_{0}+k\right\}$. We take $\varphi_{\gamma}\left(\varpi_{n}\right)$ as test function in (2.3) and using the weak convergence of the sequences $w_{n}$ and $T_{k_{0}}\left(G_{k}\left(u_{n}\right)\right)$, we get

$$
\int_{\Omega} \nabla u_{n} \cdot \nabla\left(\varpi_{n}\right) \varphi_{\gamma}^{\prime}\left(\varpi_{n}\right)+t_{n} \int_{\Omega} g\left(u_{n}\right) \frac{\left|\nabla u_{n}\right|^{2}}{1+\frac{1}{n}\left|\nabla u_{n}\right|^{2}} \varphi_{\gamma}\left(\varpi_{n}\right)=\varepsilon(n)
$$

where $\varepsilon(n)$ denotes any quantity that tends to 0 as $n$ diverges.

If we use that $g, \varphi_{\gamma}\left(k_{0}-T_{k_{0}}\left(G_{k}(u)\right)\right)$ and $\varphi_{\gamma}\left(T_{k_{0}}\left(G_{k}\left(u_{n}\right)\right)\right)$ are nonnegative and the definition of $c$, we derive the following estimate of the second term in (2.5).

$$
\begin{aligned}
\int_{\Omega} g\left(u_{n}\right) \frac{\left|\nabla u_{n}\right|^{2}}{1+\frac{1}{n}\left|\nabla u_{n}\right|^{2}} \varphi_{\gamma}\left(\varpi_{n}\right) \geq & \int_{\left\{\begin{array}{c}
k<u_{n} \leq k_{0} \\
u>k
\end{array}\right\}} \frac{g\left(u_{n}\right)\left|\nabla u_{n}\right|^{2}}{1+\frac{1}{n}\left|\nabla u_{n}\right|^{2}} \varphi_{\gamma}\left(\varpi_{n}\right) \\
& +\int_{\left\{\begin{array}{c}
u_{n} \leq k \\
u>k
\end{array}\right\}} \frac{g\left(u_{n}\right)\left|\nabla u_{n}\right|^{2}}{1+\frac{1}{n}\left|\nabla u_{n}\right|^{2}} \varphi_{\gamma}\left(\varpi_{n}\right) \\
\geq & -c \int_{\Omega}\left|\nabla T_{k_{0}}\left(G_{k}\left(u_{n}\right)\right)\right|^{2}\left|\varphi_{\gamma}\left(\varpi_{n}\right)\right| \\
& -\int_{\Omega} g\left(u_{n}\right) \frac{\left|\nabla T_{k}\left(u_{n}\right)\right|^{2}}{1+\frac{1}{n}\left|\nabla T_{k}\left(u_{n}\right)\right|^{2}} T_{k_{0}}\left(G_{k}(u)\right) .
\end{aligned}
$$

We are going to prove that the last term in the above inequality is convergent to zero as $n$ goes to infinity. Indeed, taking $T_{k_{0}}\left(G_{k}(u)\right)$ as test function in (2.3), it is 
easily deduced that

$$
\lim _{n \rightarrow \infty} \int_{\Omega} g\left(u_{n}\right) \frac{\left|\nabla u_{n}\right|^{2}}{1+\frac{1}{n}\left|\nabla u_{n}\right|^{2}} T_{k_{0}}\left(G_{k}(u)\right)=\int_{\Omega} g(u)\left|\nabla G_{k}(u)\right|^{2} T_{k_{0}}\left(G_{k}(u)\right) .
$$

On the other hand using Fatou Lemma and that $g \geq 0$ we have

$$
\begin{aligned}
\int_{\Omega} g(u)\left|\nabla G_{k}(u)\right|^{2} T_{k_{0}}\left(G_{k}(u)\right) & \leq \liminf _{n \rightarrow \infty} \int_{\Omega} g\left(u_{n}\right) \frac{\left|\nabla G_{k}\left(u_{n}\right)\right|^{2}}{1+\frac{1}{n}\left|\nabla G_{k}\left(u_{n}\right)\right|^{2}} T_{k_{0}}\left(G_{k}(u)\right) \\
& \leq \limsup _{n \rightarrow \infty} \int_{\Omega} g\left(u_{n}\right) \frac{\left|\nabla G_{k}\left(u_{n}\right)\right|^{2}}{1+\frac{1}{n}\left|\nabla G_{k}\left(u_{n}\right)\right|^{2}} T_{k_{0}}\left(G_{k}(u)\right) \\
& \leq \lim _{n \rightarrow \infty} \int_{\Omega} g\left(u_{n}\right) \frac{\left|\nabla u_{n}\right|^{2}}{1+\frac{1}{n}\left|\nabla u_{n}\right|^{2}} T_{k_{0}}\left(G_{k}(u)\right) \\
& =\int_{\Omega} g(u)\left|\nabla G_{k}(u)\right|^{2} T_{k_{0}}\left(G_{k}(u)\right),
\end{aligned}
$$

and hence we also obtain

$$
\liminf _{n \rightarrow \infty} \int_{\Omega} g\left(u_{n}\right) \frac{\left|\nabla G_{k}\left(u_{n}\right)\right|^{2}}{1+\frac{1}{n}\left|\nabla G_{k}\left(u_{n}\right)\right|^{2}} T_{k_{0}}\left(G_{k}(u)\right)=\int_{\Omega} g(u)\left|\nabla G_{k}(u)\right|^{2} T_{k_{0}}\left(G_{k}(u)\right) .
$$

Consequently, using that

$$
\begin{array}{r}
\int_{\Omega} g\left(u_{n}\right) \frac{\left|\nabla T_{k}\left(u_{n}\right)\right|^{2}}{1+\frac{1}{n}\left|\nabla T_{k}\left(u_{n}\right)\right|^{2}} T_{k_{0}}\left(G_{k}(u)\right)=\int_{\Omega} g\left(u_{n}\right) \frac{\left|\nabla u_{n}\right|^{2}}{1+\frac{1}{n}\left|\nabla u_{n}\right|^{2}} T_{k_{0}}\left(G_{k}(u)\right) \\
-\int_{\Omega} g\left(u_{n}\right) \frac{\left|\nabla G_{k}\left(u_{n}\right)\right|^{2}}{1+\frac{1}{n}\left|\nabla G_{k}\left(u_{n}\right)\right|^{2}} T_{k_{0}}\left(G_{k}(u)\right),
\end{array}
$$

we have shown that

$$
\lim _{n \rightarrow \infty} \int_{\Omega} g\left(u_{n}\right) \frac{\left|\nabla T_{k}\left(u_{n}\right)\right|^{2}}{1+\frac{1}{n}\left|\nabla T_{k}\left(u_{n}\right)\right|^{2}} T_{k_{0}}\left(G_{k}(u)\right)=0 .
$$

Using this, (2.5) and (2.6) we deduce that

$$
\int_{\Omega}\left|\nabla\left(\varpi_{n}\right)\right|^{2}\left[\alpha \varphi_{\gamma}^{\prime}\left(\varpi_{n}\right)-2 c\left|\varphi_{\gamma}\left(\varpi_{n}\right)\right|\right] \phi \leq \varepsilon(n) .
$$

Since $\gamma>c^{2}$, we have

$$
\varphi_{\gamma}^{\prime}(s)-2 c\left|\varphi_{\gamma}(s)\right| \geq \frac{1}{2}
$$

and the strong convergence of $\varpi_{n}=T_{k_{0}}\left(G_{k}\left(u_{n}\right)\right)-T_{k_{0}}\left(G_{k}(u)\right)$ to zero in $H_{0}^{1}(\Omega)$ has been established.

Step 2. $T_{k}\left(u_{n}\right)$ is strongly convergent to $T_{k}(u)$ in $H_{0}^{1}(\Omega)$. To make it, we consider the real functions $\sigma, \psi, \psi_{n}:(0,+\infty) \rightarrow \mathbb{R}$, defined by

$$
\sigma(s)=\int_{1}^{s} g(r) d r, \quad \psi(s)=\int_{0}^{s} e^{-t^{*} \sigma(r)} d r, \quad \psi_{n}(s)=\int_{0}^{s} e^{-t_{n} \sigma(r)} d r,
$$

for every $s>0$. If $\varepsilon>0,0<u, u_{n} \in H_{0}^{1}(\Omega)$ and we denote

$$
\begin{gathered}
\eta_{n, \varepsilon}=\psi_{n}\left(T_{k}\left(u_{n}\right)+\varepsilon\right)-\psi_{n}(\varepsilon)-\psi\left(T_{k}(u)+\varepsilon\right)+\psi(\varepsilon), \\
\sigma_{\varepsilon}(s)=\sigma\left(T_{k}(s)+\varepsilon\right)
\end{gathered}
$$


then the function $\varphi=e^{-t_{n} \sigma_{\varepsilon}\left(u_{n}\right)} \eta_{n, \varepsilon}$ belongs to $H_{0}^{1}(\Omega) \cap L^{\infty}(\Omega)$. Indeed, observe that $|\nabla \varphi| \in L^{2}(\Omega)$ and $|\varphi| \leq e^{-t_{n} \sigma(\varepsilon)} \eta_{n, \varepsilon} \in H_{0}^{1}(\Omega) \cap L^{\infty}(\Omega)$. Therefore, we can take $\varphi$ as test function in the problem (2.3) to deduce that

$$
\begin{gathered}
\int_{\Omega} \nabla T_{k}\left(u_{n}\right) e^{-t_{n} \sigma_{\varepsilon}\left(u_{n}\right)} \nabla \eta_{n, \varepsilon}-t_{n} \int_{\Omega} g\left(T_{k}\left(u_{n}\right)+\varepsilon\right)\left|\nabla T_{k}\left(u_{n}\right)\right|^{2} e^{-t_{n} \sigma_{\varepsilon}\left(u_{n}\right)} \eta_{n, \varepsilon} \\
+t_{n} \int_{\Omega} g\left(T_{k}\left(u_{n}\right)\right)\left|\nabla T_{k}\left(u_{n}\right)\right|^{2} e^{-t_{n} \sigma_{\varepsilon}\left(u_{n}\right)} \eta_{n, \varepsilon}=\int_{\Omega}\left(\lambda_{n}\left(w_{n}^{+}\right)^{p}+f_{0}\right) e^{-t_{n} \sigma_{\varepsilon}\left(u_{n}\right)} \eta_{n, \varepsilon} \\
-\int_{\Omega} \nabla G_{k}\left(u_{n}\right) e^{-t_{n} \sigma(k+\varepsilon)} \nabla \eta_{n, \varepsilon}-t_{n} \int_{\Omega} g\left(u_{n}\right)\left|\nabla G_{k}\left(u_{n}\right)\right|^{2} e^{-t_{n} \sigma(k+\varepsilon)} \eta_{n, \varepsilon} .
\end{gathered}
$$

Similarly, taking $\varphi=e^{-t^{*} \sigma_{\varepsilon}(u)} \eta_{n, \varepsilon}$ as test function in (2.4)

$$
\begin{aligned}
\int_{\Omega} \nabla T_{k}(u) e^{-t^{*} \sigma_{\varepsilon}(u)} \nabla \eta_{n, \varepsilon}-t^{*} \int_{\Omega} g\left(T_{k}(u)+\varepsilon\right)\left|\nabla T_{k}(u)\right|^{2} e^{-t^{*} \sigma_{\varepsilon}(u)} \eta_{n, \varepsilon} \\
+t^{*} \int_{\Omega} g\left(T_{k}(u)\right)\left|\nabla T_{k}(u)\right|^{2} e^{-t^{*} \sigma_{\varepsilon}(u)} \eta_{n, \varepsilon}=\int_{\Omega}\left(\lambda\left(w^{+}\right)^{p}+f_{0}\right) e^{-t^{*} \sigma_{\varepsilon}(u)} \eta_{n, \varepsilon} \\
\quad-\int_{\Omega} \nabla G_{k}(u) e^{-t^{*} \sigma(k+\varepsilon)} \nabla \eta_{n, \varepsilon}-t^{*} \int_{\Omega}\left|\nabla G_{k}(u)\right|^{2} g(u) e^{-t^{*} \sigma(k+\varepsilon)} \eta_{n, \varepsilon} .
\end{aligned}
$$

We are going to subtract both identities and to pass to the limit as $\varepsilon$ goes to zero. We will use that some of the resulting terms are convergent to zero. Indeed, observing that $\psi_{n}\left(T_{k}\left(u_{n}\right)\right)-\psi\left(T_{k}(u)\right)$ is bounded in $H_{0}^{1}(\Omega)$ and it has zero limit a.e. in $\Omega$, we derive that $\psi_{n}\left(T_{k}\left(u_{n}\right)\right)-\psi\left(T_{k}(u)\right)$ is weakly convergent to zero in $H_{0}^{1}(\Omega)$ and

$$
\begin{gathered}
\lim _{n \rightarrow+\infty} \int_{\Omega}\left(\lambda_{n}\left(w_{n}^{+}\right)^{p}+f_{0}\right) e^{-t_{n} \sigma\left(T_{k}\left(u_{n}\right)\right)}\left[\psi_{n}\left(T_{k}\left(u_{n}\right)\right)-\psi\left(T_{k}(u)\right)\right]=0, \\
\lim _{n \rightarrow+\infty} \int_{\Omega}\left(\lambda\left(w^{+}\right)^{p}+f_{0}\right) e^{-t^{*} \sigma\left(T_{k}(u)\right)}\left[\psi_{n}\left(T_{k}\left(u_{n}\right)\right)-\psi\left(T_{k}(u)\right)\right]=0, \\
\lim _{n \rightarrow+\infty} \int_{\Omega} \nabla G_{k}(u) e^{-t^{*} \sigma(k)} \nabla\left[\psi_{n}\left(T_{k}\left(u_{n}\right)\right)-\psi\left(T_{k}(u)\right)\right]=0 .
\end{gathered}
$$

Using again the strong convergence of $G_{k}\left(u_{n}\right)$ to $G_{k}(u)$ in $H_{0}^{1}(\Omega)$ we also have

$$
\lim _{n \rightarrow+\infty} \int_{\Omega} \nabla G_{k}\left(u_{n}\right) e^{-t_{n} \sigma(k)} \nabla\left[\psi_{n}\left(T_{k}\left(u_{n}\right)\right)-\psi\left(T_{k}(u)\right)\right]=0 .
$$

In addition, taking into account that $g(u)|\nabla u|^{2} \in L^{1}(\Omega)$ and $g\left(u_{n}\right)\left|\nabla u_{n}\right|^{2}$ is bounded in $L^{1}(\Omega)$, we deduce that

$$
\lim _{n \rightarrow+\infty} \int_{\Omega} g(u)\left|\nabla G_{k}(u)\right|^{2} e^{-t^{*} \sigma(k)}\left[\psi_{n}\left(T_{k}\left(u_{n}\right)\right)-\psi\left(T_{k}(u)\right)\right]=0
$$

and

$$
\limsup _{n \rightarrow+\infty} t_{n} \int_{\Omega} g\left(u_{n}\right)\left|\nabla G_{k}\left(u_{n}\right)\right|^{2} e^{-t_{n} \sigma(k)}\left[\psi_{n}\left(T_{k}\left(u_{n}\right)\right)-\psi\left(T_{k}(u)\right)\right] \geq 0 .
$$

Consequently, subtracting the above-mentioned identities and using the Fatou lemma, that $g$ is decreasing in a neighborhood of 0 and the above convergences we 
obtain that

$$
\int_{\Omega}\left|\nabla\left[\psi_{n}\left(T_{k}\left(u_{n}\right)\right)-\psi\left(T_{k}(u)\right)\right]\right|^{2} \leq \liminf _{\varepsilon \rightarrow 0} \int_{\Omega}\left|\nabla \eta_{n, \varepsilon}\right|^{2}=\delta_{n}
$$

with $\delta_{n}$ converging to zero. Therefore, we get

$$
\lim _{n \rightarrow+\infty} \int_{\Omega}\left|\nabla\left[\psi_{n}\left(T_{k}\left(u_{n}\right)\right)-\psi\left(T_{k}(u)\right)\right]\right|^{2}=0 .
$$

Taking into account that

$$
\begin{aligned}
\nabla\left[T_{k}\left(u_{n}\right)-T_{k}(u)\right]= & e^{t_{n} \sigma\left(T_{k}\left(u_{n}\right)\right)} \nabla\left[\psi_{n}\left(T_{k}\left(u_{n}\right)\right)-\psi\left(T_{k}(u)\right)\right] \\
& -\left[e^{-t_{n} \sigma\left(T_{k}\left(u_{n}\right)\right)}-e^{-t^{*} \sigma\left(T_{k}(u)\right)}\right] \nabla T_{k}(u) e^{t_{n} \sigma\left(T_{k}\left(u_{n}\right)\right)}
\end{aligned}
$$

and noting that, as $n$ goes to infinity, $e^{-t_{n} \sigma\left(T_{k}\left(u_{n}\right)\right)}-e^{-t^{*} \sigma\left(T_{k}(u)\right)}$ converges to 0 in $L^{2}(\Omega)$ and that $e^{t_{n} \sigma\left(T_{k}\left(u_{n}\right)\right)}$ is bounded in $L^{\infty}(\Omega)$, we deduce that $T_{k}\left(u_{n}\right)$ converges strongly in $H_{0}^{1}(\Omega)$ to $T_{k}(u)$ and Step 2 has been proved. As it has been observed, this suffices to conclude also that $u_{n}$ converges strongly in $H_{0}^{1}(\Omega)$ to $u$.

Remark 2.7. Notice that the $H_{0}^{1}(\Omega)$ estimate proved in the proof does not depend on the function $g$.

Remark 2.8. With the same proof, the previous proposition remains true even if we replace $f_{0}$ in $(2.3)$ by any sequence of data $f_{n}$ strongly convergent to $f_{0}$ in $L^{2 N / N+2}(\Omega)$.

\section{Global COntinua of solutions}

As it has been mentioned in the Introduction, we apply the Leray-Schauder degree to obtain existence of "continua of solutions" of (1.1), i.e. connected and closed subsets in the solution set

$$
S=\left\{(\lambda, u) \in \mathbb{R} \times H_{0}^{1}(\Omega): u=K_{\lambda}(u)\right\} .
$$

The first result of this section is related to the case $f_{0} \gtreqless 0$ and it states the existence of global continua in the solution set $S$ emanating from the unique solution of (1.1) for $\lambda=0$.

Theorem 3.1. Consider $0 \leqq f_{0} \in L^{\frac{2 N}{N+2}}(\Omega)$ and assume that hypothesis $(G)$ holds. Then there exists an unbounded continuum $\Sigma \subset S$ of positive solutions which contains $\left(0, u_{0}\right)$, where $u_{0}$ is the unique solution of (1.1) for $\lambda=0$.

Proof. First, we compute the index of the solution $u_{0} \in H_{0}^{1}(\Omega)$ for (1.1) with $\lambda=0$. Remind that for every isolated solution $u_{\lambda} \in H_{0}^{1}(\Omega)$ of (1.1) for some $\lambda \in \mathbb{R}$, we denote by $i\left(K_{\lambda}, u_{\lambda}\right)$ the index of such a solution, that is, the topological LeraySchauder degree $\operatorname{deg}\left(I-K_{\lambda}, B_{\varepsilon}\left(u_{\lambda}\right), 0\right)$ of the operator $I-K_{\lambda}$ in a ball $B_{\varepsilon}\left(u_{\lambda}\right)$ centered at $u_{\lambda}$ with radius $\varepsilon>0$ small enough (to assure that $u_{\lambda}$ is the unique solution of (1.1) in this ball).

We claim that $i\left(K_{0}, u_{0}\right)=1$. To prove the claim we denote by $U(t)$ the unique solution for

$$
\begin{cases}-\Delta u+\operatorname{tg}(u)|\nabla u|^{2}=f_{0}(x) & \text { in } \Omega, \\ u=0 & \text { on } \partial \Omega,\end{cases}
$$

and we define $H:[0,1] \times H_{0}^{1}(\Omega) \rightarrow H_{0}^{1}(\Omega)$ by $H(t, w)=U(t)$ for every $(t, w) \in$ $[0,1] \times H_{0}^{1}(\Omega)$. Observe that $H(1, w)=U(1)=K_{0}(w)=u_{0}$, while $H(0, w)=$ 
$U(0)=(-\Delta)^{-1}\left(f_{0}(x)\right)$ and it is well known that $i\left((-\Delta)^{-1}\left(f_{0}(x)\right), U(0)\right)=1$. By Proposition 2.5 we deduce that $H$ is compact. In addition, as it has been pointed out in Remark 2.7, taking $R=\mathcal{S}\left\|f_{0}\right\|_{2 N /(N+2)}>0$, we have $\|U(t)\|<R$, for every $t \in[0,1]$. Hence, $u \neq H(t, u)$ for every $t \in[0,1]$ and $u \in H_{0}^{1}(\Omega)$ with $\|u\| \geq R$ and we can apply the homotopy invariance of the degree to conclude that

$$
\begin{aligned}
i\left(K_{0}, u_{0}\right) & =i(H(1, \cdot), U(1))=i(H(0, \cdot), U(0)) \\
& =i\left((-\Delta)^{-1}\left(f_{0}(x)\right), U(0)\right)=1,
\end{aligned}
$$

and the claim has been proved. The theorem follows now from the Rabinowitz Theorem 3.2 in [18] (see also [16]).

Remark 3.2. The keystone to compute the index $i\left(K_{0}, u_{0}\right)$ in the above proof has been to show that the problem (1.1) with $\lambda=0$ is homotopic to the one-dimensional $(t \geq 0)$ family of problems (3.1), which for $t=0$ is nothing but a linear problem. Some remarks can be added about this problem (3.1). If for every $t>0$ we denote by $u(t)$ the unique solution of it, we immediately deduce that the set

$$
\Sigma_{0}=\left\{(t, u(t)) \in[0,+\infty) \times H_{0}^{1}(\Omega)\right\}
$$

is an unbounded continuum. In addition, if $f_{0} \in L^{r}(\Omega)$ for some $r>\frac{N}{2}$, then, taking $G_{k}(u(t))$ as test function, by Stampacchia technique [20], we obtain the existence of a positive constant $C>0$ not depending neither on $n$ nor $t$, such that $\|u(t)\|_{\infty} \leq C$. Hence the projection $\operatorname{Proj}_{[0,+\infty)} \Sigma_{0}$ of $\Sigma_{0}$ on the $t$-axis is all the interval $[0,+\infty)$ which guarantees the unboundedness of $\Sigma_{0}$. Furthermore, if $\inf _{s \in(0, C)} g(s)>0$, we claim that $\lim _{t \rightarrow+\infty}\|u(t)\|=0$. Indeed, choosing $\frac{T_{\varepsilon}(u(t))}{\varepsilon}$ as test function, we obtain

$$
\frac{1}{\varepsilon} \int_{\{u(t) \leq \varepsilon\}}|\nabla u(t)|^{2}+t \int_{\Omega} g(u(t))|\nabla u(t)|^{2} \frac{T_{\varepsilon}(u(t))}{\varepsilon}=\int_{\Omega} f_{0} \frac{T_{\varepsilon}(u(t))}{\varepsilon} .
$$

In particular,

$$
t \int_{\Omega} g(u(t))|\nabla u(t)|^{2} \frac{T_{\varepsilon}(u(t))}{\varepsilon} \leq \int_{\Omega} f_{0} .
$$

Taking now limits as $\varepsilon$ goes to zero and using Fatou Lemma, we get

$$
t \inf _{s \in(0, C)} g(s)\|u(t)\|^{2} \leq t \int_{\Omega} g(u(t))|\nabla u(t)|^{2} \leq \int_{\Omega} f_{0},
$$

from which the claim is clearly deduced.

In the case that $g$ is continuous in a neighborhood of zero and $f_{0} \equiv 0$ (which implies that the unique solution $u_{0}$ of (1.1) is the trivial one $u_{0} \equiv 0$ ) there is as in Theorem 3.1, a continuum of (trivial) solutions emanating from $(0,0)$. We prove now that bifurcation from the line of trivial solutions occurs provided that, in addition, $0 \leq p \leq 1$. Moreover, the bifurcation point is

$$
\mu^{*}= \begin{cases}0, & \text { if } 0 \leq p<1, \\ \mu_{1}, & \text { if } p=1,\end{cases}
$$

where we remind that $\mu_{1}$ is denoting the first eigenvalue of the Laplacian operator with zero Dirichlet boundary conditions. In the sequel, $\phi_{1}$ denotes the corresponding first positive eigenfunction associated to $\mu_{1}$ with norm in $H_{0}^{1}(\Omega)$ equal to one. 
Theorem 3.3. Assume that $g:[0,+\infty) \rightarrow[0,+\infty)$ is continuous, $f_{0} \equiv 0$ and $0 \leq p \leq 1$. Then $\mu^{*}$ is the only bifurcation point from zero of positive solution for (1.1). Moreover, there exists an unbounded continuum $\Sigma_{0} \subset S$ of positive solutions emanating from $\left(\mu^{*}, 0\right)$.

In order to prove Theorem 3.3 we use the global bifurcation theorem by Rabinowitz (see again [18]) or, more specifically, that the index of the (isolated) trivial solution changes as $\lambda$ crosses $\lambda=\mu^{*}$. This will be a direct consequence of the following two lemmas.

Lemma 3.4. If $\Lambda \subset\left(-\infty, \mu^{*}\right)$ is a bounded closed interval, then there exists $\varepsilon>0$ such that for every $\lambda \in \Lambda$ and every $t \in[0,1]$ problem

$$
\begin{cases}-\Delta u+t g\left(u^{+}\right)|\nabla u|^{2}=t \lambda^{+}\left(u^{+}\right)^{p} & \text { in } \Omega, \\ u=0 & \text { on } \partial \Omega,\end{cases}
$$

admits no solution $u \in H_{0}^{1}(\Omega)$ with $\|u\| \in(0, \varepsilon]$.

Proof. First, in the case $0 \leq p<1$ (thus $\mu^{*}=0$ ) and $\Lambda \subset(-\infty, 0)$, the result holds for any $\varepsilon>0$ since the unique solution of (3.2) for $\lambda<0$ is the trivial one.

Assume now that $p=1$, that is $\mu^{*}=\mu_{1}$. We prove the assertion of the lemma by contradiction. Assume that there exist sequences $0 \leq \lambda_{n} \in \Lambda, t_{n} \in[0,1]$ and $0 \leq u_{n} \in H_{0}^{1}(\Omega)$ with $0<\left\|u_{n}\right\| \rightarrow 0$, such that

$$
\begin{cases}-\Delta u_{n}+t_{n} g\left(u_{n}\right)\left|\nabla u_{n}\right|^{2}=t_{n} \lambda_{n} u_{n} & \text { in } \Omega, \\ u_{n}=0 & \text { on } \partial \Omega .\end{cases}
$$

Observe that $\int_{0}^{u_{n}} e^{-t_{n} \int_{1}^{s} g(t) d t} d s=w_{n} \in H_{0}^{1}(\Omega)$. We denote by $z_{n}$ the sequence $z_{n}=\frac{w_{n}}{\left\|w_{n}\right\|}$. Up to a subsequence, $z_{n}$ weakly converges to $z \in H_{0}^{1}(\Omega)$ and the convergence is strongly in $L^{q}(\Omega)$ for every $q<2^{*}$.

Using Lemma 2.3 we can take

$$
\varphi=\frac{e^{-t_{n} \int_{1}^{u_{n}} g(t) d t}\left(z_{n}-z\right)}{\left\|w_{n}\right\|}
$$

as test function in (3.2). Thus, we have

$$
\int_{\Omega} \nabla z_{n} \nabla\left(z_{n}-z\right)=t_{n} \lambda_{n} \int_{\Omega} z_{n} \frac{u_{n} e^{-t_{n} \int_{1}^{u_{n}} g(t) d t}}{\int_{0}^{u_{n}} e^{-t_{n} \int_{1}^{s} g(t) d t} d s}\left(z_{n}-z\right) .
$$

By the dominated convergence theorem we obtain the convergence $\int_{\Omega} \nabla z_{n} \nabla\left(z_{n}-\right.$ $z) \rightarrow 0$. Therefore we deduce that $z_{n} \rightarrow z$ in $H_{0}^{1}(\Omega)$ and, in particular, $\|z\|=1$.

On the other hand, using Lema 2.3 , we can take $\varphi=\frac{e^{-t_{n} \int_{1}^{u_{n}} g(t) d t} \phi_{1}}{\left\|w_{n}\right\|}$ as test function. This leads to

$$
\int_{\Omega} \nabla z_{n} \nabla \phi_{1}=t_{n} \lambda_{n} \int_{\Omega} z_{n} \frac{u_{n} e^{-t_{n} \int_{1}^{u_{n}} g(t) d t}}{\int_{0}^{u_{n}} e^{-t_{n} \int_{1}^{s} g(t) d t} d s} \phi_{1}
$$

Using that $\left\|u_{n}\right\|_{\infty} \rightarrow 0$ (by Lemma 2.3) and passing to the limit by dominated convergence theorem we obtain, up to a subsequence, that $t_{n} \rightarrow t^{*}, \lambda_{n} \rightarrow \lambda<$ 
$\mu_{1}=\mu^{*}$ and

$$
\mu_{1} \int_{\Omega} z \phi_{1}=\int_{\Omega} \nabla z \nabla \phi_{1}=t^{*} \lambda \int_{\Omega} z \phi_{1} .
$$

Taking into account that $t^{*} \lambda<\mu_{1}$, the only possibility in the previous inequality is $z=0$. This is a contradiction with $\|z\|=1$ and therefore the lemma has been also proved in the case $p=1$.

Lemma 3.5. For every bounded closed interval $\Lambda \subset\left(\mu^{*}, \infty\right)$, there exists $\varepsilon>0$ such that if $t \in[0,1]$ and $\lambda \in \Lambda$, then problem

$$
\begin{cases}-\Delta u+g(u)|\nabla u|^{2}=\lambda u^{p}+t & \text { in } \Omega, \\ u=0 & \text { on } \partial \Omega,\end{cases}
$$

admits no positive solution $u \in H_{0}^{1}(\Omega)$ with $\|u\| \in(0, \varepsilon]$.

Proof. Observe that if $u \in H_{0}^{1}(\Omega)$ is a solution of (3.4) and we choose (by Lemma 2.3) $\varphi=e^{-\int_{1}^{u} g(t) d t} \phi_{1}$ as test function, then we obtain

$$
\int_{\Omega} e^{-\int_{1}^{u} g(t) d t} \nabla u \nabla \phi_{1}=\int_{\Omega}\left(\lambda u^{p}+t\right) e^{-\int_{1}^{u} g(t) d t} \phi_{1} .
$$

Noting also that $\int_{0}^{u} e^{-\int_{1}^{s} g(t) d t} d s=w \in H_{0}^{1}(\Omega)$, we can rewrite the above identity as

$$
\int_{\Omega} \nabla w \nabla \phi_{1}=\lambda \int_{\Omega} w \frac{u^{p} e^{-\int_{1}^{u} g(t) d t}}{\int_{0}^{u} e^{-\int_{1}^{s} g(t) d t} d s} \phi_{1}+t \int_{\Omega} w \frac{e^{-\int_{1}^{u} g(t) d t}}{\int_{0}^{u} e^{-\int_{1}^{s} g(t) d t} d s} \phi_{1} .
$$

We divide now the rest of our proof in cases according to the value $p$. Case 1: $p<1\left(\mu^{*}=0\right)$. Using that $\frac{s^{p}}{\int_{0}^{s} e^{-\int_{1}^{t} g(u) d u} d t} \geq s^{p-1} e^{-\int_{0}^{1} g(u) d u}$, for every $s \geq 0$, we have

$$
\lim _{s \rightarrow 0^{+}} \frac{s^{p}}{\int_{0}^{s} e^{-\int_{1}^{t} g(u) d u} d t}=+\infty
$$

and, if $\lambda_{0}=\min \Lambda$, there exists $s_{0} \in(0,1)$ such that

$$
\frac{s^{p}}{\int_{0}^{s} e^{-\int_{1}^{r} g(t) d t} d r}>\frac{\mu_{1}}{\lambda_{0}}+1, \quad \forall s \in\left(0, s_{0}\right) .
$$

We claim that, if $\lambda \in \Lambda$, problem (3.4) has no solutions $u \in H_{0}^{1}(\Omega)$ with norm $\|u\| \leq\left(\frac{s_{0}}{\alpha}\right)^{1 / t}$, where $\alpha$ is given by (2.2). Indeed, if by contradiction, we assume the existence of such a solution $u$ for some $\lambda \in \Lambda$, then, by Lemma $2.3\|u\|_{\infty} \leq s_{0}<1$. Consequently, we obtain

$$
\frac{u^{p}}{\int_{0}^{u} e^{-\int_{1}^{r} g(t) d t} d r}>\frac{\mu_{1}}{\lambda_{0}}+1
$$

and

$$
e^{-\int_{1}^{u} g(t) d t} \geq 1
$$

This, jointly to (3.5) and the definition of $\mu_{1}$ implies that

$$
\mu_{1} \int_{\Omega} w \phi_{1} \geq \lambda\left(\frac{\mu_{1}}{\lambda_{0}}+1\right) \int_{\Omega} w \phi_{1},
$$

which is a contradiction $\left(\lambda \geq \lambda_{0}\right)$ proving the claim and thus the lemma in the case $p<1$. 
Case 2: $p=1$. It suffices to show that if, for some $\lambda>0$, there exist sequences $t_{n} \in[0,1], \lambda_{n} \rightarrow \lambda$ and $0 \leq u_{n} \in H_{0}^{1}(\Omega)$ with $0<\left\|u_{n}\right\| \rightarrow 0$, such that

$$
\begin{cases}-\Delta u_{n}+g\left(u_{n}\right)\left|\nabla u_{n}\right|^{2}=\lambda_{n} u_{n}+t_{n} & \text { in } \Omega, \\ u_{n}=0 & \text { on } \partial \Omega,\end{cases}
$$

then $\lambda \leq \mu^{*}=\mu_{1}$. To make it, denote $w_{n}=\int_{0}^{u_{n}} e^{-\int_{1}^{s} g(t) d t} d s$ and $z_{n}=$ $w_{n} /\left\|w_{n}\right\|$. We can assume that, up to a subsequence, $z_{n}$ converges weakly in $H_{0}^{1}(\Omega)$ and strongly in $L^{q}(\Omega)$ for every $q<2^{*}$ to some $z \in H_{0}^{1}(\Omega)$. Taking $\varphi=e^{-\int_{1}^{u_{n}} g(t) d t}\left(z_{n}-z\right) /\left\|w_{n}\right\|$ as test function in (3.6), we deduce that

$$
\begin{aligned}
\int_{\Omega} \nabla z_{n} \nabla\left(z_{n}-z\right)= & \lambda_{n} \int_{\Omega} z_{n} \frac{u_{n} e^{-\int_{1}^{u_{n}} g(t) d t}}{\int_{0}^{u_{n}} e^{-\int_{1}^{s} g(t) d t} d s}\left(z_{n}-z\right) \\
& +\frac{t_{n}}{\left\|w_{n}\right\|} \int_{\Omega} e^{-\int_{1}^{u_{n}} g(t) d t}\left(z_{n}-z\right),
\end{aligned}
$$

and, by dominated convergence theorem, that

$$
\lim _{n \rightarrow \infty} \int_{\Omega} \nabla z_{n} \nabla\left(z_{n}-z\right)=0 .
$$

Therefore, $z_{n} \rightarrow z$ in $H_{0}^{1}(\Omega)$ and $\|z\|=1$.

On the other hand, dividing (3.5) (with $u=u_{n}$ and $t=t_{n}$ ) by $\left\|w_{n}\right\|$, we have

$$
\begin{aligned}
\int_{\Omega} \nabla z_{n} \nabla \phi_{1}-\lambda_{n} \int_{\Omega} z_{n} \frac{u_{n} e^{-\int_{1}^{u_{n}} g(t) d t}}{\int_{0}^{u_{n}} e^{-\int_{1}^{s} g(t) d t} d s} \phi_{1} & =\frac{t_{n}}{\left\|w_{n}\right\|} \int_{\Omega} e^{-\int_{1}^{u_{n}} g(t) d t} \phi_{1} \\
& \geq 0 .
\end{aligned}
$$

Using that, by Lemma $2.3,\left\|u_{n}\right\|_{\infty} \rightarrow 0$ and passing to the limit we deduce that

$$
0 \leq \int_{\Omega} \nabla z \nabla \phi_{1}-\lambda \int_{\Omega} z \phi_{1}=\left(\mu_{1}-\lambda\right) \int_{\Omega} z \phi_{1} .
$$

Since $z \neq 0$, we get $\lambda \leq \mu_{1}$ and the proof of the lemma is completed also in the case $p=1$.

Remark 3.6. Observe that a direct consequence of the previous two lemmas is that $\mu^{*}$ is the only possible bifurcation point from zero for positive solutions of (1.1).

Proof of Theorem 3.3 completed. We claim that $i\left(K_{\lambda}, 0\right)=1$ for every $\lambda<\mu^{*}$ and that $i\left(K_{\lambda}, 0\right)=0$ for every $\lambda>\mu^{*}$. In order to do that we use the above two lemmas and the homotopy invariance of the degree.

If $\lambda<\mu^{*}$, we define the operator $H:[0,1] \times H_{0}^{1}(\Omega) \rightarrow H_{0}^{1}(\Omega)$ by taking $H(t, w)$ as the unique solution $u \in H_{0}^{1}(\Omega)$ of the problem

$$
\begin{gathered}
-\Delta u+t g(u)|\nabla u|^{2}=t \lambda^{+}\left(w^{+}\right)^{p}, \quad x \in \Omega, \\
u=0, \quad x \in \partial \Omega .
\end{gathered}
$$

By Lemma 3.4 with $\Lambda=\{\lambda\}$, we can consider $\varepsilon>0$ such that $u \neq H(t, u)$ for every $t \in[0,1]$ and $u \in H_{0}^{1}(\Omega)$ with $\|u\|=\varepsilon$. Since $H$ is compact by Proposition 2.5, we can apply the homotopy invariance of the degree to conclude that

$$
\begin{aligned}
i\left(K_{\lambda}, 0\right) & =\operatorname{deg}\left(I-H(1, \cdot), B_{\varepsilon}(0), 0\right) \\
& =\operatorname{deg}\left(I-H(0, \cdot), B_{\varepsilon}(0), 0\right)=i\left((-\Delta)^{-1}, 0\right)=1 .
\end{aligned}
$$


On the other hand, if $\lambda>\mu^{*}$ we can apply in this case the Lemma 3.5 to choose $\varepsilon>0$ such that if $\widetilde{H}(t, w)$ is now the unique solution of

$$
\begin{gathered}
-\Delta u+\operatorname{tg}(u)|\nabla u|^{2}=\lambda\left(w^{+}\right)^{p}+t, \quad x \in \Omega, \\
u=0, \quad x \in \partial \Omega,
\end{gathered}
$$

where $t \in[0,1]$ and $w \in H_{0}^{1}(\Omega)$, then $u \neq \widetilde{H}(t, u)$ for every $t \in[0,1]$ and $u \in H_{0}^{1}(\Omega)$ with $\|u\|=\varepsilon$. Applying again the homotopy invariance of the degree to the compact (by Remark (2.8)) operator $\widetilde{H}:[0,1] \times H_{0}^{1}(\Omega) \rightarrow H_{0}^{1}(\Omega)$, we obtain

$$
i\left(K_{\lambda}, 0\right)=\operatorname{deg}\left(I-\widetilde{H}(0, \cdot), B_{\varepsilon}(0), 0\right)=\operatorname{deg}\left(I-\widetilde{H}(1, \cdot), B_{\varepsilon}(0), 0\right)=0,
$$

where the computation of the last degree is due to Lemma 3.5 which implies that $u \neq \widetilde{H}(1, u)$ for every $u \in B_{\varepsilon}(0)$.

Thus, we have proved the change of index if $\lambda$ crosses $\lambda=\mu^{*}$ and the proof follows by using the Rabinowitz Global Bifurcation Theorem.

\section{EXISTENCE FOR LARGE $\lambda$}

The unboundedness of the continuum $\Sigma$ obtained in Theorem 3.1 implies that one of the projections of $\Sigma$, either its projection $\operatorname{Proj}_{H_{0}^{1}(\Omega)} \Sigma$ on the $H_{0}^{1}(\Omega)$-axis or its projection $\operatorname{Proj}_{[0,+\infty)} \Sigma$ on the $\lambda$-axis, is an unbounded set. In this section we give sufficient conditions to assure that $\operatorname{Proj}_{[0,+\infty)} \Sigma$ is unbounded. The role of these conditions is to provide for every compact set $\Lambda$ of $\lambda$ 's the existence of suitable a priori bounds of the $H_{0}^{1}(\Omega)$-norm of solutions of (1.1) with $\lambda \in \Lambda$, i.e., to establish that $\operatorname{Proj}_{H_{0}^{1}(\Omega)}\left[\Sigma \cap\left(\Lambda \times H_{0}^{1}(\Omega)\right)\right]$ is bounded. These a priori bounds result is independent from the existence of $\Sigma$, more precisely no condition at zero for $g$ is required. The arguments also apply for the continuum $\Sigma_{0}$ given by Theorem 3.3 .

Theorem 4.1. The projection $\operatorname{Proj}_{[0,+\infty)} \Sigma$ (respectively, $\operatorname{Proj}_{[0,+\infty)} \Sigma_{0}$ ) of the continuum $\Sigma$ (respectively, $\Sigma_{0}$ ) given by Theorem 3.1 (respectively, by Theorem 3.3) on the $\lambda$-axis is unbounded provided that, in addition to the hypotheses of those theorems, one of the following conditions is satisfied:

i) either $0 \leq p<1$,

ii) or $1 \leq p<2$ and $g$ verifies (1.4) for some constants $s_{1}, c>0$ and $\gamma<2-p$. Moreover, if in addition $g$ satisfies that

$$
g(s) \leq C\left(s^{q}+\frac{1}{s}\right), \quad \forall s>0
$$

for some $q \leq p$ and $0 \lesseqgtr f_{0}$, given a sequence $\left(\lambda_{n}, u_{n}\right)$ in $\Sigma$ (respectively, in $\Sigma_{0}$ ) with $\lambda_{n} \rightarrow+\infty$ then $\left\|u_{n}\right\| \rightarrow+\infty$.

Proof. We just give the details of the proof for the continuum $\Sigma$. The proof for the continuum $\Sigma_{0}$ is equal with the only change of $\Sigma$ by $\Sigma_{0}$. As it has been mentioned, either $\operatorname{Proj}_{H_{0}^{1}(\Omega)} \Sigma$ or $\operatorname{Proj}_{[0,+\infty)} \Sigma$ is an unbounded set. To prove the theorem, it suffices to show that $\operatorname{Proj}_{H_{0}^{1}(\Omega)}\left[\Sigma \cap\left(\Lambda \times H_{0}^{1}(\Omega)\right)\right]$ is bounded for every compact $\Lambda \subset \mathbb{R}$, i.e. the norm $\|u\|$ of every solution of (1.1) is bounded provided that $\lambda$ is in a bounded set. Indeed, this implies that if $\operatorname{Proj}_{H_{0}^{1}(\Omega)} \Sigma$ is unbounded, then we also get the unboundedness of $\operatorname{Proj}_{[0,+\infty)} \Sigma$. To make it, we take a positive solution $u \in H_{0}^{1}(\Omega)$ of (1.1) for some $\lambda>0$. We divide the proof in two parts according as i) or ii) holds. 
i) In the case $0 \leq p<1$, taking $u /\|u\|^{p+1}$ as test function and setting $z=\frac{u}{\|u\|}$, we obtain

$$
\begin{aligned}
\|u\|^{1-p} & \leq\|u\|^{-p-1} \int_{\Omega}\left(|\nabla u|^{2}+g(u)|\nabla u|^{2} u\right) \\
& \leq \lambda \int_{\Omega} z^{p+1}+\int \frac{f_{0} z}{\|u\|^{p}} .
\end{aligned}
$$

Using Hölder and Sobolev inequalities

$$
\begin{aligned}
\|u\|^{1-p} & \leq \lambda\|z\|_{2^{*}}^{p+1}|\Omega|^{1-\frac{p+1}{2^{*}}}+\frac{1}{\|u\|^{p}}\left\|f_{0}\right\|_{\frac{2 N}{N+2}}\|z\|_{2^{*}} \\
& \leq \mathcal{S}^{p+1} \lambda|\Omega|^{1-\frac{p+1}{2^{*}}}+\frac{\mathcal{S}}{\|u\|^{p}}\left\|f_{0}\right\|_{\frac{2 N}{N+2}},
\end{aligned}
$$

which proves in this case that $\|u\|$ is bounded as $\lambda$ is bounded.

ii) Let us consider the case $1 \leq p<2$ and assume that (1.4) holds with $\gamma<2-p$. Thanks to this condition, we may construct a continuous and non negative function $h(s)$ such that $h(s)=0$ for every $s<\frac{s_{0}}{2}, h(s)=\frac{c}{s^{\gamma}}$ for every $s>s_{0}$ and $g(s) \geq h(s)$ for every $s>0$. We also define the function $\varphi(s)$ given by

$$
\varphi(s)=\int_{0}^{s} \exp \left(-\int_{v}^{s} h(t) d t\right) d v, \quad \forall s \geq 0 .
$$

It is elementary to prove that

(a) $0 \leq \varphi(s) \leq s$ for every $s \in(0,+\infty)$.

(b) $\varphi^{\prime}(s)+h(s) \varphi(s)=1$ for every $s \in(0,+\infty)$.

(c) There exits $\sigma>0$ with $h(s) \varphi(s) \leq \sigma$ for every $s>0$.

Observe that, using (c), $h(u) \varphi(u) \in L^{\infty}(\Omega)$, which, by (b), implies that $\nabla \varphi(u) \in L^{2}(\Omega)$. Taking into account that, by (a), we have $\varphi(u) \leq u$ we deduce that $\varphi(u) \in H_{0}^{1}(\Omega)$. Hence we can take $\varphi(u)$ as test function and using that $h(s) \leq g(s)$ we obtain

$$
\begin{aligned}
\|u\|^{2} & \stackrel{(b)}{=} \int_{\Omega}|\nabla u|^{2}\left(\varphi^{\prime}(u)+h(u) \varphi(u)\right) \\
& \leq \int_{\Omega}\left(\nabla u \nabla \varphi(u)+g(u)|\nabla u|^{2} \varphi(u)\right) \\
& =\int_{\Omega}\left(\lambda u^{p}+f_{0}\right) \varphi(u) .
\end{aligned}
$$

Observe also that $\frac{\varphi(s)}{s^{\gamma}}$ is bounded from above near infinity (by (c) and construction of $h$ ) and near zero (by definition of $\varphi$ and $\gamma<2-p<1$ ). Thus, there exists $C>0$ such that $\varphi(s) \leq C s^{\gamma}$ for every $s>0$. Therefore, dividing by $\|u\|^{p+\gamma}$ in the previous inequality and using the Hölder and Sobolev inequalities as before we get

$$
\begin{aligned}
\|u\|^{2-p-\gamma} & \leq C \lambda \int_{\Omega} z^{p+\gamma}+\int \frac{f_{0} z}{\|u\|^{p+\gamma-1}} \\
& \leq C \lambda \mathcal{S}^{p+\gamma}|\Omega|^{1-\frac{p+\gamma}{2^{*}}}+\frac{\mathcal{S}}{\|u\|^{p+\gamma-1}}\left\|f_{0}\right\|_{2 N /(N+2)},
\end{aligned}
$$

and consequently, we deduce again that if $\lambda$ is bounded, then the norm $\|u\|$ is also bounded. 
In conclusion, it has been proved the unboundedness of $\operatorname{Proj}_{[0,+\infty)} \Sigma$ and the first part of theorem is concluded.

Now, we assume that $0 \lesseqgtr f_{0}$ and we prove the second part of the theorem by contrapositive. Indeed, if $\left(\lambda_{n}, u_{n}\right) \in \Sigma$, then considering $0 \leq \varphi \in C_{c}^{\infty}(\Omega)$ and taking $\frac{\varphi}{u_{n}^{q}}$ as test function (it is an admissible test function thanks to Proposition 2.4 ) we have

$$
\begin{aligned}
\int_{\Omega} \nabla u_{n} \frac{\nabla \varphi}{u_{n}^{q}}-q \int_{\Omega} \frac{\left|\nabla u_{n}\right|^{2}}{u_{n}^{q+1}} \varphi+\int_{\Omega} \frac{g\left(u_{n}\right)}{u_{n}^{q}}\left|\nabla u_{n}\right|^{2} \varphi & \\
-\int_{\Omega} f_{0} \frac{\varphi}{u_{n}^{q}} & =\lambda_{n} \int_{\Omega} u_{n}^{p-q} \varphi .
\end{aligned}
$$

Dropping negative terms we get

$$
\int_{\Omega} \nabla u_{n} \frac{\nabla \varphi}{u_{n}^{q}}+\int_{\Omega} \frac{g\left(u_{n}\right)}{u_{n}^{q}}\left|\nabla u_{n}\right|^{2} \varphi \geq \lambda_{n} \int_{\Omega} u_{n}^{p-q} \varphi
$$

By Proposition 2.4, $u_{n}$ is uniformly away from zero in $\operatorname{supp} \varphi$ and therefore, using (4.1) we deduce that $\frac{g\left(u_{n}\right)}{u_{n}^{q}}$ is bounded from above in $\operatorname{supp} \varphi$ and the sequence $\int_{\Omega} u_{n}^{p-q} \varphi$ is also away from zero. Therefore, if $u_{n}$ is bounded in $H_{0}^{1}(\Omega)$, the lefthand side of the above equality is bounded from above and then $\lambda_{n}$ has to be also bounded. We have proved the contrapositive of the second part of theorem.

Remark 4.2. Actually, if $\lambda>0$ is fixed, we have proved in the previous theorem an a priori estimate for the $H_{0}^{1}(\Omega)$-norm of solutions of (1.1). This a priori bound is the usual keystone to prove the existence of solutions by means of an approximating approach. We have to point out that this is not enough, at least, in the case of singular functions $g$ where the uniform strong maximum principle is also essential. For instance, if $u \in H_{0}^{1}(\Omega)$ is a solution of the problem

$$
\begin{cases}-\Delta u+\frac{|\nabla u|^{2}}{u^{2+\varepsilon}}=1 & \text { in } \Omega, \\ u=0 & \text { on } \partial \Omega,\end{cases}
$$

and we take $u$ as test function, we obtain a priori estimates of the norm $\|u\|$. However, it has been proved in [5] that this problem has no solution for $\varepsilon>0$.

\section{Nonexistence For LARge $\lambda$}

In the semilinear case $(g \equiv 0)$ it is known that, given $f_{0} \geqq 0$ and $p>1$, there exists $\lambda^{*}>0$ such that (1.1) has no positive solution for every $\lambda>\lambda^{*}$. Notice also that $\lambda^{*}=\mu_{1}$ in the case $p=1$ even if $f_{0} \equiv 0$. As it is shown in the previous section, the quasilinear case is quite different. However, this section is devoted to obtain sufficient conditions on $g$ for the existence of such a $\lambda^{*}$.

Theorem 5.1. Consider $0 \lesseqgtr f_{0} \in L^{\frac{2 N}{N+2}}(\Omega)$ and $g \geq 0$ a continuous function in $(0,+\infty)$. Assume also that either $g$ is continuous at zero or $\sqrt{g}$ is decreasing and integrable in a neighborhood of zero with $\lim _{s \rightarrow 0} g(s)=+\infty$. If condition (1.5) holds and there exists $c_{1}, c_{2} \geq 0$ such that

$$
\exp \left(-2 \int_{1}^{s} g(t) d t\right) \leq c_{1} g(s)+c_{2}, \quad \forall s<1,
$$


then, there exists $\lambda^{*}>0$ such that (1.1) has no positive solution for $\lambda>\lambda^{*}$.

Remark 5.2. If $g$ is integrable in a neighborhood of zero, then (5.1) is trivially satisfied. Therefore if, in addition to (1.5), hypothesis $(\mathrm{G})$ is satisfied then the unbounded continuum $\Sigma$ given by Theorem 3.1 satisfies $\operatorname{Proj}_{[0,+\infty} \Sigma \subset\left[0, \lambda^{*}\right]$.

Proof. For $\omega \subset \subset \Omega$ we denote by $\chi_{\omega}(x)$ the charactheristic function of $\omega$ and consider the first eigenvalue (respectively eigenfunction) $\mu_{\omega}$ (respectively $\phi_{\omega}$ ) associated to the eigenvalue problem

$$
\begin{cases}-\Delta u=\lambda \chi_{\omega}(x) u & \text { in } \Omega, \\ u=0 & \text { on } \partial \Omega .\end{cases}
$$

It suffices to show that a necessary condition for the existence of solution $u \in$ $H_{0}^{1}(\Omega)$ of $(1.1)$ is $\mu_{\omega} \geq \lambda c$, for a suitable positive constant $c$. To make it, consider a sequence of functions $0 \leq \phi_{n} \in C_{c}^{\infty}(\Omega)$ converging in $H_{0}^{1}(\Omega)$ to $\phi_{\omega}$. Observe that the function $\varphi(u)=e^{-\int_{1}^{T_{k}(u)} g(t) d t} \phi_{n}$ belongs to $H_{0}^{1}(\Omega) \cap L^{\infty}(\Omega)$ (use Proposition 2.4 in the case that $g$ is unbounded near zero). Thus, taking $\varphi(u)$ as test function in the equation satisfied by $u$ and using that $f_{0} \geq 0$ we get

$$
\begin{array}{r}
\int_{\Omega} \nabla u \nabla \phi_{n} e^{-\int_{1}^{T_{k}(u)} g(t) d t}+\int_{\{u \geq k\}} g(u)|\nabla u|^{2} e^{-\int_{1}^{k} g(t) d t} \phi_{n} \\
\geq \lambda \int_{\Omega} u^{p} e^{-\int_{1}^{T_{k}(u)} g(t) d t} \phi_{n} .
\end{array}
$$

Taking limits, firstly as $k$ tends to $\infty$ (using Fatou Lemma) and secondly as $n$ goes to $\infty$ (using the $H_{0}^{1}(\Omega)$-convergence of $\phi_{n}$ to $\phi_{\omega}$ and Lebesgue Theorem by $(5.1)$ ), we have

$$
\int_{\Omega} \nabla u \nabla \phi_{\omega} e^{-\int_{1}^{u} g(t) d t} \geq \lambda \int_{\Omega} u^{p} e^{-\int_{1}^{u} g(t) d t} \phi_{\omega} .
$$

We claim now that the function $w=\int_{0}^{u} e^{-\int_{1}^{s} g(t) d t} d s$ belongs to $H_{0}^{1}(\Omega)$. Indeed, using (5.1) and that $\sqrt{g}$ is integrable at zero, we have

$$
\begin{aligned}
0 \leq w & =\int_{0}^{1} e^{-\int_{1}^{s} g(t) d t} d s+\int_{1}^{u} e^{-\int_{1}^{s} g(t) d t} d s \\
& \leq \int_{0}^{1} \sqrt{c_{1} g(s)+c_{2}} d s+u-1 \in L^{2}(\Omega) .
\end{aligned}
$$

On the other hand by the definition of solution, $g(u)|\nabla u|^{2} \in L^{1}(\Omega)$ and by (5.1) we deduce that $|\nabla u|^{2} e^{-2 \int_{1}^{u} g(t) d t} \chi_{\{u<1\}} \leq|\nabla u|^{2}\left(c_{1} g(u)+c_{2}\right) \in L^{1}(\Omega)$. Thus,

$$
\nabla w=\nabla u e^{-\int_{1}^{u} g(t) d t} \chi_{\{u<1\}}+\nabla u e^{-\int_{1}^{u} g(t) d t} \chi_{\{u \geq 1\}} \in L^{2}(\Omega) .
$$

Now, using again (5.1) and that $\sqrt{g} \in L^{1}(0,1)$, we obtain $\int_{0}^{1} e^{-\int_{1}^{s} g(t) d t} d s<\infty$ and we can define the function $\psi(u)=\frac{u^{p} e^{-\int_{1}^{u} g(t) d t}}{\int_{0}^{u} e^{-\int_{1}^{s} g(t) d t} d s}$ for $u>0$. Therefore,

$$
\begin{aligned}
\mu_{\omega} \int_{\Omega} \chi_{\omega}(x) w \phi_{\omega} & =\int_{\Omega} \nabla w \nabla \phi_{\omega} \geq \lambda \int_{\Omega} w \phi_{\omega} \psi(u) \\
& \geq \lambda \int_{\Omega} \chi_{\omega}(x) w \phi_{\omega} \psi(u)
\end{aligned}
$$


Using Proposition 2.4, there exists $L_{\omega}>0$ such that $u(x)>L_{\omega}$ a.e. $x \in \omega$. Moreover, by condition (1.5), $c:=\inf _{s \in\left[c_{\omega}, \infty\right)} \psi(s)>0$ and we conclude that

$$
\mu_{\omega} \int_{\Omega} w \phi_{\omega} \geq \lambda c \int_{\Omega} w \phi_{\omega}
$$

that is, $\mu_{\omega} \geq \lambda c$ as desired.

\section{Proofs of the Main theorems}

We show in this section how to prove Theorems 1.1, 1.2, 1.3 and 1.4 from the results in the previous sections.

Proof of Theorem 1.1. We deduce from Theorem 3.1 the existence of an unbounded continuum $\Sigma \subset S$ of positive solutions which contains $\left(0, u_{0}\right)$, where $u_{0}$ is the unique solution of (1.1) for $\lambda=0$.

(1) In the case that (1.3) holds, we use Theorem 5.1 to assure that there exists $\lambda^{*}>0$ such that (1.1) has no positive solutions for $\lambda>\lambda^{*}$.

(2) If (1.4) is satisfied, according to Theorem 4.1 the projection $\operatorname{Proj}_{[0,+\infty)} \Sigma$ on the $\lambda$-axis is unbounded.

Proof of Theorem 1.2. According to Theorem 3.1, there exists an unbounded continuum $\Sigma \subset S$ which contains $\left(0, u_{0}\right)$, where $u_{0}$ is the unique solution of (1.1) for $\lambda=0$. Moreover, thanks to Theorem 4.1, the projection $\operatorname{Proj}_{[0,+\infty)} \Sigma_{0}$ on the $\lambda$-axis is unbounded.

Proof of Theorem 1.3. By Theorem 3.1, there exists an unbounded continuum $\Sigma \subset S$ which contains $\left(0, u_{0}\right)$, where $u_{0}$ is the unique solution of (1.1) for $\lambda=0$. The rest of the proof falls naturally into two parts according to the statement of the theorem.

(1) If (1.4) is satisfied, by Theorem 4.1, the projection $\operatorname{Proj}_{[0,+\infty)} \Sigma_{0}$ on the $\lambda$-axis is unbounded.

(2) If (1.5) holds, using Theorem 5.1, it follows that there exists $\lambda^{*}>0$ such that (1.1) has no positive solutions for $\lambda>\lambda^{*}$.

Proof of Theorem 1.4.

(1)-(2) In order to prove items (1) and (2) we first apply Theorem 3.3 to get the existence of an unbounded continuum $\Sigma_{0} \subset S$ of positive solutions emanating from $\left(\mu_{1}, 0\right)$. If $(1.3)$ is satisfied (i.e. item (1)) we argue as in the proof of Theorem 5.1. Observe that if $f_{0} \equiv 0$, USMP is no longer true. However, the function $\psi$ defined in the proof of Theorem 5.1 satisfies $c=\inf _{s \in(0, \infty)} \psi(s)>0$. Hence, we can deal with the same proof with $\omega=\Omega$ to obtain again that there exists $\lambda^{*}>0$ such that (1.1) has no positive solutions for $\lambda>\lambda^{*}$ and item (1) follows. On the other hand, if (1.4) is verified then, thanks to Theorem 4.1, the projection $\operatorname{Proj}_{[0,+\infty)} \Sigma_{0}$ on the $\lambda$-axis is unbounded and we finish the proof of item (2).

(3) We use again Theorem 3.3 to deduce the existence of an unbounded continuum $\Sigma_{0} \subset S$ of positive solutions emanating from $(0,0)$. We conclude from Theorem 4.1 that the projection $\operatorname{Proj}_{[0,+\infty)} \Sigma_{0}$ on the $\lambda$-axis is unbounded.

(4) We define the function

$$
\varphi(s)=\int_{0}^{T_{1}(s)} \exp \left(\int_{s}^{t} g(r) d r\right) d t, \quad \forall s>0,
$$


which satisfies the following.

(a) $\varphi^{\prime}(s)+g(s) \varphi(s)=\left[T_{1}^{\prime}(s)\right]^{2}$ for every $0<s \neq 1$.

(b) There exists a positive constant $C$ such that $s^{p} \varphi(s) \leq C\left[T_{1}(s)\right]^{2}$. Indeed, this is trivial for $s \leq 1$, while for $s>1$, taking into account that $h \leq 0$,

$$
\begin{aligned}
s^{p} \varphi(s) & =\int_{0}^{1} s^{p} \exp \left(\int_{s}^{1} g(r) d r+\int_{1}^{t} g(r) d r\right) d t \\
& =\varphi(1) \exp \left(\int_{s}^{1}\left(g(r)-\frac{p}{r}\right) d r\right) \\
(1.6) & \leq \varphi(1) \exp \left(-\int_{1}^{+\infty} h(r) d r\right) \equiv C \leq C\left[T_{1}(s)\right]^{2} .
\end{aligned}
$$

(c) If $u \in H_{0}^{1}(\Omega)$ is a positive solution of (1.1) with $p>1, f_{0} \equiv 0$ and $g$ continuous at zero, then $\varphi(u) \in H_{0}^{1}(\Omega)$. Taking $\varphi(u)$ as test function, in the equation satisfied by $\mathrm{u}$, we obtain

$$
\begin{aligned}
\mu_{1} \int_{\Omega}\left[T_{1}(u)\right]^{2} & \leq \int_{\Omega}\left|\nabla T_{1}(u)\right|^{2}=\int_{\Omega}|\nabla u|^{2}\left(\varphi^{\prime}(u)+g(u) \varphi(u)\right) \\
& \leq \lambda \int_{\Omega} u^{p} \varphi(u) \leq C \lambda \int_{\Omega}\left[T_{1}(u)\right]^{2} .
\end{aligned}
$$

We finish the proof by taking $\lambda^{*}=\frac{\mu_{1}}{C}$.

\section{REFERENCES}

[1] B. Abdellaoui, I. Peral and A. Primo, Elliptic problems with a Hardy potential and critical growth in the gradient: Non-resonance and blow-up results, J. Differential Equations, 239 (2007) 386-416.

[2] F. Andreu, L. Boccardo, L. Orsina and S. Segura de León, Existence results for $L^{1}$ data of some quasi-linear parabolic problems with a quadratic gradient term and source. Math. Models Methods Appl. Sci. 12 (2002) 1-16, DOI: 10.1142/S0218202502001520.

[3] F. Andreu, S. Segura de León and J. Toledo, Quasi-linear difusion equations with gradient terms and $L^{1}$ data. Nonlinear Analysis 56 (2004) 1175-1209.

[4] D. Arcoya and L. Boccardo, Critical points for multiple integral of Calculus of Variations. Arch. Rat. Mech. Anal. 134 (1996), 249-274.

[5] D. Arcoya, J. Carmona, T. Leonori, P.J. Martínez-Aparicio, L. Orsina and F. Petitta, Existence and nonexistence of solutions for singular quadratic quasilinear equations. J. Differential Equations 246 (2009), 4006-4042.

[6] D. Arcoya and S. Segura de León, Uniqueness of solutions for some elliptic equations with a quadratic gradient term. ESAIM Control Optim. Calc. Var., (2008) DOI: 10.1051/cocv:2008072 .

[7] A. Bensoussan, L. Boccardo and F. Murat, On a nonlinear P.D.E. having natural growth terms and unbounded solutions, Ann. Inst. H. Poincaré Anal. Non Linéaire, 5 (1988), 347-364.

[8] L. Boccardo, Dirichlet problems with singular and quadratic gradient lower order terms, ESAIM Control Optim. Calc. Var., 14 (2008) 411-426 .

[9] L. Boccardo and T. Gallouët, Nonlinear elliptic and parabolic equations involving measure data, J. Funct. Anal., 87 (1989), 149-169.

[10] L. Boccardo, T. Gallouët and F. Murat, A unified presentation of two existence results for problems with natural growth, Progress in partial differential equations: the Metz surveys, 2 (1992), 127-137, Pitman Res. Notes Math. Ser., 296, Longman Sci. Tech., Harlow, 1993.

[11] L. Boccardo and F. Murat, Almost everywhere convergence of the gradients of solutions to elliptic and parabolic equations, Nonlinear Anal., 19 (1992), 581-597. 
[12] L. Boccardo, F. Murat and J.-P. Puel, Existence de solutions non bornees pour certaines équations quasi-linéaires, Portugaliae Mathematica, 41 (1982), 507-534.

[13] L. Boccardo, F. Murat and J.-P. Puel, Existence de solutions faibles pour des équations elliptiques quasi-linéaires à croissance quadratique, Nonlinear partial differential equations and their applications. Collège de France Seminar, Vol. IV (Paris, 1981/1982), 19-73, Res. Notes in Math., 84, Pitman, Boston, 1983.

[14] L. Boccardo, L. Orsina and M.M. Porzio, Existence results for quasilinear elliptic and parabolic problems with quadratic gradient terms and sources, in preparation.

[15] A. Canino, Multiplicity of solutions for quasilinear elliptic equations, Topol. Methods Nonlinear Anal. 6 (1995), 357-370.

[16] J. Leray and J. Schauder, Topologie et équations fonctionnelles, Annales Scientifiques de l'É.N.S, 51 (1934), 45-78.

[17] L. Orsina and J-P. Puel, Positive solutions for a class of nonlinear elliptic problems involving quasilinear and semilinear terms, Commun. Partial Differential Equations, 26, 1665-1689 (2001).

[18] P.H. Rabinowitz, Some global results for nonlinear eigenvalue problems, J. Funct. Anal. 7 (1971), 487-513.

[19] D. Ruiz and A. Suárez, Existence and uniqueness of positive solution of a logistic equation with nonlinear gradient term, Proc. Roy. Soc. Edinburgh Sect. A 137 (2007), 555-566.

[20] G. Stampacchia, Equations elliptiques du second ordre à coefficients discontinuos. Les Presses de l'Université de Montréal, Montréal 1966.

(D. Arcoya) Departamento de Análisis Matemático, Campus Fuentenueva S/N, UniverSidad de Granada 18071 - Granada, Spain. e-mail: Darcoya@ugr.es

(J. Carmona) Departamento de Álgebra y Análisis Matemático, Universidad de Almería, Ctra. Sacramento s/n, La Cañada de San Urbano, 04120 - Almería, Spain. e-mail: JCARMONA@UAL.ES

(P. J. Martínez-Aparicio) Departamento de Análisis Matemático, Campus Fuentenueva S/N, Universidad de Granada 18071 - Granada, Spain. e-mail: pedrojma@ugr.es 